\title{
Exploration of the Fusion Display of ECDIS and Radar Image Information in High Latitude Sea Area
}

\author{
Shuhao Zhang ${ }^{1, \text { a }}$,Xin Liu ${ }^{2, b}$, Ning Zhang ${ }^{3, c}$ \\ ${ }^{1}$ Shandong jiaotong University Maritime College, Weihai, 264200, China \\ ${ }^{2}$ Shandong jiaotong University Maritime College, Weihai, 264200, China \\ ${ }^{3}$ Shandong jiaotong University Maritime College, Weihai, 264200, China \\ aemail: zshao2008@163.com, bemail: axinzaixian@163.com, cemail: 1241310547 @qq.com
}

Keywords: ECDIS; radar; information superimposed

\begin{abstract}
The inconsistency between the radar image information and electronic chart information in the high latitudes can result in much difficulty on the identification of marks, which will be dangerous to the ship's navigation. By analyzing the characteristics of the projection methods of the high-latitude sea area charts, we discuss the causes for the errors of the radar image and superimposed ECDIS information. This thesis puts forward the method of subdivision of longitude in the weft to correct the Mercator projection distortion, and therefore we can get the Mercator projection which is able to meet any predetermined accuracy requirements. Based on GPS coordinates value, radar real-time location information is changed into the screen coordinates through a coordinate transformation, and we can position radar center position on the ECDIS chart accurately. In this way, we can realize the real-time, rapid, precise overlay display of radar echo and ECDIS chart.
\end{abstract}

\section{Introduction}

ECDIS refers to a navigation chart information system which have sufficient reserve configuration, and can be deemed to comply with the latest amended SOLAS 1974 regulation V/19 and V/27 requirements. As a ship's mandatory instrument ECDIS are gradually completed from 2012 to 2018 when ECDIS will be an important part of the ship navigation equipments and will be of great significance to the ships' navigation[1]. Shipborne radar is the ship's important navigation equipment as well, and the obtained information can also be showed on the ECDIS display so as to make ECDIS become an integrated display system. In this way, ECDIS can not only display all the static information on the chart, and can also display static and dynamic information obtained by radar in order to achieve a synthetic display of various observation data.

The information obtained by the radar overlapping display on ECDIS. It can effectively improve the ship's ability of collision avoidance, navigation precision, correction on radar image, and can also detect GPS accuracy and help to judge the types of hazards[2].

For the comprehensive information displayed on ECDIS to achieve the above advantages, it requires the static and dynamic targets that the radar detects should superimpose accurately with the static graphs on the charts. What's more, we need to ensure that the static radar target should coincide with the targets on the charts, and the display error must be less than one pixel[3].

\section{The display of charts in ECDIS}

1.Charts projection and its property

We often employ three projection methods on charts. They are the Mercator projection, Gauss projection and gnomonic projection.

Mercator projection is an equiangle right cylindrical projection. It is a projection process of making a cylindrical surface tangent to the spherical surface of the Earth, and making the cylinder axis coincide with the earth's axis, and then projecting the earth's latitude and longitude lines onto the cylindrical surface, and then cutting the cylinder along its generatrix, and at last unfolding it to 
get a plane graph. Mercator chart has the following characteristics:

(1) Meridian lines in chart are north-south parallel straight lines, and latitude lines are east-west parallel straight lines, and the meridian and latitude lines are perpendicular to each other.

(2) The longitude 1' is of equal length, but the latitude 1's length becomes long gradually with latitude increased, and there is latitude growing phenomenon.

(3) Rhumb line on the chart is a straight line.

(4) It has the characteristics of the equiangle projection.

(5) At the same latitude the local scale is equal, whereas on different latitudes the local scale increases with latitude increases.

Gauss projection is an equiangle transverse cylindrical projection, is a projection process of using a cylindrical surface to tangent to the spherical surface of the earth, and making the cylinder axis perpendicular to the axis, and then projecting the latitude and longitude lines of the earth onto the cylindrical surface, and then cutting the cylindrical along its generatrix, and at last unfolding it to get a plane graph. It has the following characteristics:

(1) the shaft meridian is tangent to the cylindrical surface, and shaft meridian and the equator are projected as a straight line perpendicular. All other latitude and longitude lines are projected into the curves, and the farther they are from the shaft meridian and the equator, the more serious of bending deformation they become.

(2) on the same line of latitude, the length deformation increases with the increasing longitude; the shaft meridian does not have distortion. On the same line of longitude, the length of the deformation increases with the decreasing latitude, and at the equator it is the maximum.

Gnomonic projection's perspective point is in the center, and it is a method of projecting things on the surface to a surface that is tangent to the spherical earth. It has the following characteristics:

(1) The straight line on the chart represents a large circular arc, and it is the shortest route between two points of the sphere.

(2) Gnomonic projector is not an equiangle projection, so we can't measure the distance and azimuth on the chart.

2. Projection mode selection on high-latitude sea area Maintaining the Integrity of the Specifications

In the case of using chart, in order to facilitate drawing rhumb line route and bearing line on charts, navigational charts generally should meet the following two conditions: (1) on the chart rhumb line is a straight line; (2) the nature of projection is equiangle. As the Mercator chart is an equiangle right cylindrical projection it meets the above requirements, and becomes the main body of the navigational chart. Equiangle characteristics of the Mercator projection make it maintain the same shape (the ground differential circular remains the circular after projecting on chart), although the length is deformed. Therefore, it can still be used at any latitude, but due to the presence of growing meridian, larger deformation will appear when employed at high latitudes.

Although Gauss projection is equiangle projection, after the projection all other meridian and weft except the shaft meridian and the equator are curves on the chart, thus the rhumb line is curve. Consequently, it's not suitable for small scale charts to display, but while describing below the middle and low latitudes and a small area near the shaft meridian, the bending of the latitude and longitude lines are minimal. It is even less than the measurement and drawing error, as a result it can be considered to be a straight line. Secondly, in the Gauss projection, projection surface is tangent with north and south poles, therefore, it can also be used to describe the polar charts. But the deformations of meridian and weft lines have been very huge and rhumb line is not a straight line, so it is not suitable to be used as navigational chart.

Gnomonic projection uses straight line as a big arc, and it is often used to display the small scale chart. It's deformation of the length and angles are all large, so generally it is only used to make Gnomonic chart.

Taking all these into consideration, we can choose Mercator projection to display the chart in high latitude sea area. 


\section{Radars information display}

Radar utilizes the reflection phenomenon of the radio waves of the targets to discover the position of the target. When the radar detected the target, it will extract the relevant information from the target echo, and the distance plus the spatial orientation of the target can be obtained. The change rate of the target position can be obtained from the regularity of its distance and orientation's changes with time, thereby we can establish the tracking of the target. Therefore, modern radar can measure not only the direction and the distance of the target, but also the speed of the target, and other information of the target.

1. Radar target distance's measurement

When radar works, the transmitter antenna launches a string of periodically repeated high-frequency pulses to the space. If the time of the radar transmitter pulse between the radar antenna and the target is $\Delta \mathrm{t}$, the velocity of propagation of radio waves in the space is $\mathrm{C}$ (about $3 \times 10^{8} \mathrm{~m} / \mathrm{s}$ ), and R stands for the distance of the target:

$$
R=C \times \frac{\Delta t}{2}
$$

So if the target exists in the path of radio pulse propagation, then the radar may receive the echo of the target, and display it on the radar screen. The position from any maritime targets to the vessel on the radar screen can corresponds accurately to the location from the target display location on the screen to the center of the echo images region, and we can measure the distance between the target and the ship with measurement tool.

2. Measurements radar target azimuth

The radar antenna is directional circular scan antenna, and in the horizontal plane, the width of the antenna radiation is only about $1^{\circ}$. Therefore, for a certain moment, radar can only emit in one direction, and can receive the echo only in this direction[1]. The radar antenna employs the bow as the azimuth reference in the air, ring $360^{\circ}$ rotating. Radar azimuth scan system can record the rotational orientation of the antenna with respect to the bow accurately in the memory, and in accordance with the requirements of the display, it can read the data from the memory, and display the data on the screen.

After measuring the distance and azimuth of the target, the radar will display the target on the radar screen with reference to the ship's position.

\section{Overlay radar images on the ECDIS}

Combination display of charts, the ship's position and radar image of targets around the ship can provide comprehensive information for the safe navigation to the duty officer. We should convert the actual geographic coordinates to screen coordinates when display electronic chart. As a result, the Earth's surface image turns into a flat image and deformation is inevitable. Radar target is displayed on the radar screen according to the minified scale and superimposed on ECDIS, which also cause deformation while comparing the target echo images detected by radar with the real target.

\section{Error Analysis}

Electronic chart is different from radar data on coordinate system as well as display algorithm, which is the main reason for the superimposed error.

In the high latitudes, regardless of the chart projection method, there will be a relatively large error. Chart is obtained by the elliptic curved surface of the earth projected onto the plane in accordance with a certain mathematical method. And deformation is inevitable in the process of projection. With the Mercator chart, no deformation appears in the reference latitude. The farther it is away from the reference latitude, the greater the deformation becomes. At the same time, due to the presence of meridian grew phenomenon, the meridian distance between the latitude becomes longer with increasing latitude. It is nearly impossible to continuously achieve this change when drawing chart, therefore the higher the latitude, the larger the error[4].

It will also produce error when the real number rectangular coordinates convert to screen 
coordinates[4]. It can be obtained through the analysis and research that the smaller the pixel dots size on the screen, the smaller the error. Therefore, we must use a high-resolution display in order to improve the accuracy of the electronic chart display, so that we can reduce the size of the screen pixels dots[2].

\section{Error correction and Match}

Through the above analysis, we must correct the deformation of the Mercator projection in order to improve the accuracy of the electronic chart display, so that we can make it meet any predetermined precision requirements of the charts projection.

In displaying charts, we had better display chart information in detail because the deformation of the Mercator chart is relevant to the reference latitude. However, the complexity of calculation can not be too much can it should be able to respond quickly to the display speed. As a consequence it is more convenient to segment the longitude in the latitude. We must obey regular features of meridian grow when the meridian line segments the latitude. First we estimate a range represented as a difference of latitude interval, and the error between a subdivision of the interval and the interval according to the meridian growing subdivision should not be greater than $0.1 \mathrm{~mm}$, which is the drawing error. Then we can calculate the ordinate at both ends of the partition value according to the interval of the interval subdivision calculation. Interval subdivision can be evenly divided. This will not only ensure the accuracy of the subdivision calculation, but also avoid excess calculation in order to meet the predetermined requirements.

To convert the error of the real number rectangular coordinates to screen coordinates, we should use a high-resolution display to reduce the size of the screen pixels dots.

For the radar images to be superimposed on the electronic chart the electronic chart should be shown first. Based on the two characteristics of the Mercator projection, we can choose the ship's latitude as the reference latitude, and we can not only quickly display electronic charts, but also reduce the deformation within the electronic chart display area. Then according to the GPS coordinates, we can change the radar real-time location information by coordinate transformation when displaying the chart to get the screen coordinates (ie, the actual geographic coordinates $\rightarrow$ Mercator projection coordinates $\rightarrow$ screen coordinates), thus we can locate the radar center position on the chart accurately. Then we can calculate the radar image scale by radar search limits and radar video image size[5]. And we can see the radar image on chart according to the central location, scaling and selected charts. In this way we can realize the real-time, rapid and precise overlay display between the radar echo video and the electronic chart.

\section{Conclusion}

Radar is utilized in coastal waters, especially in the fog, night navigation and collision avoidance. Radar has great advantages, but the simple radar position fixing causes it out of connected between the target measurement and the chart work, and we can not acquire intuitive ship's position only from the radar. Therefore, the superimposed display between the electronic chart and radar images is the inevitable requirement for the development of radar navigation, and it is also the inevitable trend of function expansion of electronic chart. Taking electronic chart as display layer, we can analyze and correct the length deformation of the electronic chart, locate the position of the radar center on the chart accurately[5]. As a consequence, we can realize the real-time, rapid and precise overlay display between the radar echo video and the electronic chart so that the static radar target coincides with the target in the charts correspondingly, and we can realize the real-time accurate positioning of the dynamic radar target on the chart.

\section{Acknowledgement}

In this paper, the research was sponsored by Shandong Jiaotong University Science and Technology Research Fundation(Project NO.Z201102). 


\section{References}

[1] Wenjun Zhang. Navigation[M]. Dalian Maritime University Press. 2012.8.P239.

[2] Yanfeng Liang. ECDIS/Radar Image Overlay Software Technology Research[D].Harbin Engineering University. 2010.

[3] Wu Qing. The research on the overlay of radar image and chart graph in the electronic chart system[J].JIANGSU SHIP.2004.

[4] Yingjun Zhang ect.edited. The basic mathematics and algorithms of electronic chart. Dalian. Dalian Maritime University Press.2000.8.

[5] GongLiu Yang, YuBao Dou and RongCai Zheng.Method of Image Overlay on Radar and Electronic Chart[J]. Journal of Chinese Inertial Technology.2010.04. 